\title{
Implementación de un chatbot para la comunicación con los clientes del sistema de agua y/0 alcantarillado administrado por empresa SEDAPAL S.A.
}

\author{
Implementation of a chatbot for communication \\ with customers of the water and / or sewerage \\ system managed by the company SEDAPAL S.A.
}

\author{
iD ${ }^{1}$ Marco Antonio Salcedo Huarcayas (iD) 'Sebastian Sanchez Diaz (iD) 'Nancy Isabel Herrera Paico \\ (iD ${ }^{2} J o r g e$ Rafael Diaz Dumont
}

Universidad César Vallejo. Lima, Perú.

Universidad Nacional Autónoma de Tayacaja "Daniel Hernández Morillo". Pampas, Perú. ${ }^{2}$

\section{Resumen}

El Servicio de Agua Potable y Alcantarillado de Lima -SEDAPAL,es la más grande empresa pública que ofrece el servicio de agua potable y alcantarillado en el Perú, pero viene afrontando una serie de limitaciones asociadas a la comunicación con los clientes referido a problemas operativos que están generando reclamos, causando costos por ello; una de las causas es la falta de nuevos canales de comunicación vinculados a la relación cliente-empresa, como las redes sociales, mensajería, microblogging y apoyados en nuevas estrategias como Chatbot, esta es la propuesta de esta investigación. Se consideró el chatbot porque está dentro del alcance del marketing conversacional y trae como beneficios un servicio 24 horas, respuestas instantáneas, quejas resueltas rápidamente, respuestas detalladas, buena interacción, entre otros; además utiliza la Comprensión del Lenguaje Natural (NLU, Natural Language Understanding) que permite a la máquina pueda comprender y actuar eficientemente.El Retorno de la Inversión - ROI, de esta propuesta permitiría ahorrar \$5,007.57 dólares anualmente, pudiendo recuperar toda la inversión en el sexto año. Según Gartner, llegará el momento en que las personas se comuniquen con las empresas con el mismo feeling como lo hacen con otras personas.

Palabras clave: SEDAPAL, problemas operativos, chatbots, Comprensión del Lenguaje Natural.

Recibido: Enero 2020

Aceptado: Mayo 2020

\section{Abstract}

The Drinking Water and Sewerage Service of Lima - SEDAPAL, is the largest public company that offers drinking water and sewerage service in Peru, but it has been facing a series of limitations associated with communication with customers regarding operational problems that they are generating claims, causing costs for it; One of the causes is the lack of new communication channels linked to the customer-company relationship, such as social networks, messaging, microblogging and supported by new strategies such as Chatbot, this is the proposal of this research. The chatbot was considered because it is within the scope of Con- 
versational Marketing and brings as benefits a 24-hour service, instant responses, complaints quickly resolved, detailed responses, good interaction, among others; It also uses the Natural Language Understanding (NLU) that allows the machine to understand and act efficiently. The Return on Investment - ROI, from this proposal would save $\$ 5,007.57$ dollars annually, being able to recover all the investment in the sixth year. According to Gartner, the time will come when people communicate with companies with the same feeling as they do with other people.

Keywords: SEDAPAL, operational problems, chatbots, Natural Language Comprehension.

\section{INTRODUCCIÓN}

En el Perú existen 50 Empresas que prestan el servicio de Saneamiento; de las cuales, SEDAPAL(2017) según "el Informe de Sostenibilidad 2017 es la mayor empresa ya que cuenta con más de 1,5 millonesde conexiones de agua, se estima que representa a 7 millones de usuarios. En otras zonas, son administrados por las municipalidades" (p. 11).

SEDAPAL (2016), es "una empresa estatal de derecho privado constituida como sociedad anónima y tiene por finalidad la prestación eficiente de los servicios de saneamiento, dotando de agua potable y alcantarillado sanitario a la ciudad de Lima y Callao, cumpliendo para ello con una serie de actividades, entre otros: i) atención de emergencias por fugas de agua, filtraciones, atoros, red, buzones represados, atención de aniegos; ii) control y regulación de las válvulas por operación, mantenimiento, y abastecimiento de sistemas de agua potable, iii) supervisión de los servicios prestados por terceros, expedientes de reclamos, recepción de obras, verificando el cumplimiento de procesos constructivos; iv) gestionar adecuadamente las conexiones y factibilidades de conexiones de agua y alcantarillado, proyectos de inversión, supervisión y recepción de obras; y, v) atendiendo las necesidades y gestión de carácter administrativo, en los Centros de servicios correspondientes, así como en La Atarjea" (p. s/n).

Para este artículo, el tema propuesto está relacionado a las mejoras en la comunicación con el cliente que debe realizar los Equipos Operativos denominados en la empresa como Equipos Operación y Mantenimiento de redes, que funcionalmente dependen de la Gerencia de Servicios Norte, Centro y Sur, siendo necesario conocer las actividades que en ella se desarrolla.

\section{Problema de la investigación}

El problema principal se enfoca en la deficiente comunicación con el clientereferido a actividades operativas, en la cual se originadiversos reclamos recibidos hacia la empresa. Es de conocimiento que, según los usuarios, las fallas en las que incurre la empresa son las siguientes: a) falta de reporte de fallas en el sistema de red de agua y/o alcantarillado al cliente, b) ausencia de una comunicación adecuada en relación a los cierres y reaperturas de servicios, c) desinformación sobre el horario de abastecimiento de la zona, etc.

Las falencias principales de esta empresa son: a) Los procedimientos operativos requieren ser creados, actualizados, revisados y/o estandarizados, b) Los recursos asignados a la comunicación con el cliente son insuficientes, c) No existe una plataforma tecnológica CustomerRelationship Management CRM, que se encuentre integrado con los demás sistemas, d) Las aplicaciones orientadas a una arquitectura web son insuficientes, lo que no facilita el desarrollo de soluciones CRM alineadas con tendencias tecnológicas, etc.

\section{Indicadores de insatisfacción para el cliente}

Según la memoria anual 2018 de SEDAPAL viene afrontando una serie de problemas por mantener el alcantarillado y las diversas redes de agua de manera óptima. 


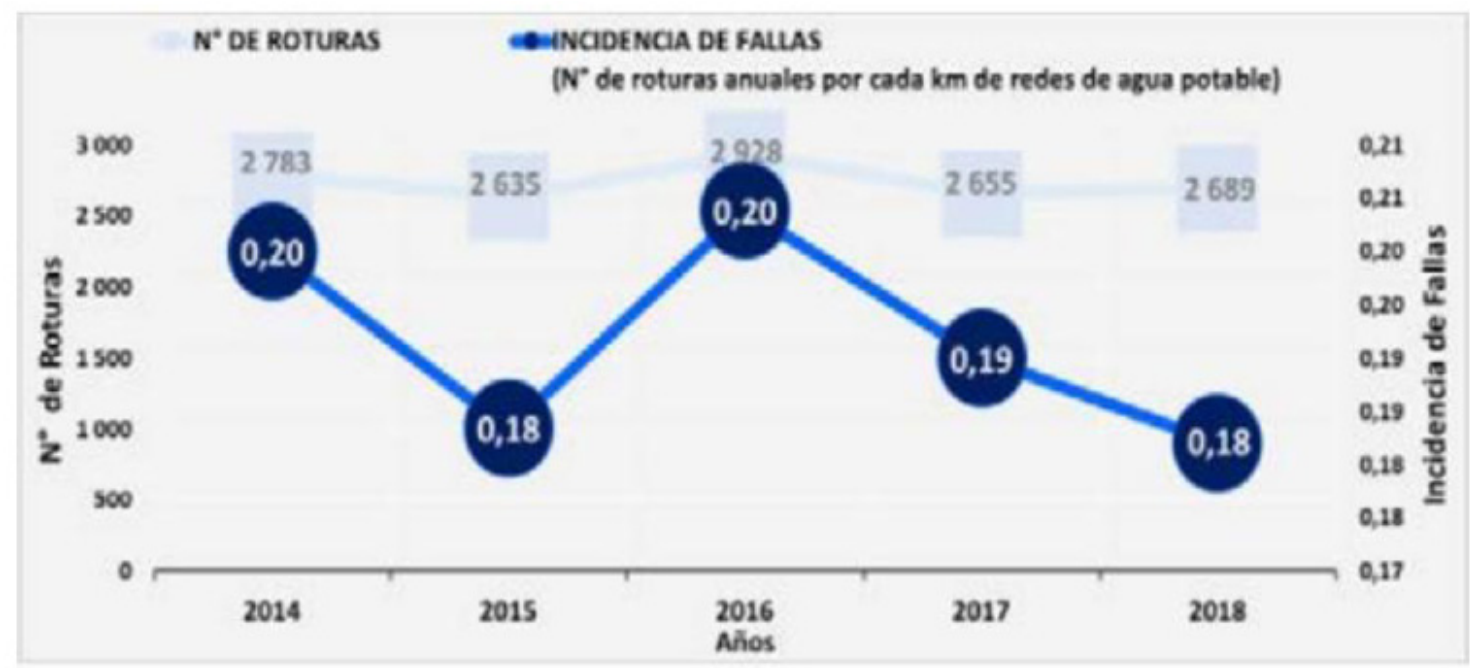

Figura 1. Incidencia de fallas en redes de agua potable (en $\mathrm{N}^{\circ}$ de roturas por $\mathrm{km}$ ) Fuente: Memoria Anual 2018.

\section{Estudio de nivel de satisfacción al cliente}

Según Pastor (2018)"el Informe de encuesta realizada por la empresa CCR en el 2017 a 2958 personas, en el ámbito de Lima y Callao, a personas de 25 a 60 años de los Niveles Socio-Económico $A, B, C$ y $D$, que son usuarios de SEDAPAL con suministro de tipo doméstico, se identificaron problemas operativos en el servicio de agua y alcantarillado; identificándose el $41 \%$ no se le ha comunicado los cortes programados del servicio de agua, siendo el mantenimiento de tuberías el de mayor incidencia 76\%, luego la limpieza de tanques, entre otros" (p. s/n).
Todos estos hechos han generado los mismos clientes soliciten información a través de los diversos canales "tradicionales"como Aquafono, en Centros de Atención, o página de internet.Pero, sin embargo, no se ha encontrado una solución concisa y eficaz a los problemas, generando que se conviertan en reclamos.

\section{Descripción del proceso de reclamos}

Los reclamos se inician con la recepción, luego por la investigación, conciliación y finalmente la respuesta.

Tabla 1. Reclamos por el servicio, roturas

\begin{tabular}{|c|c|c|c|c|}
\hline Conceptos & Unidad & 2015 & $\begin{array}{l}\text { Años } \\
2016\end{array}$ & 2017 \\
\hline \multicolumn{5}{|c|}{ Actividades que generaron reclamo por falta de gestión con el cliente } \\
\hline $\begin{array}{l}\text { Roturas en redes } \\
\text { primarias y } \\
\text { secundarias }\end{array}$ & $\mathrm{N}^{\circ}$ Roturas & 2,635 & 2,928 & 2,978 \\
\hline
\end{tabular}

Fuente: Adaptado del Anuario Estadístico (2016 y 2017), Estadísticos Comerciales de SEDAPAL y el sistema comercial OPEN SGC. 
Las causas del incremento de reclamos operativos son (Bradbury, 2007, p.s/n):

- "Falta de un Plan basado en CRM para la gestión operativa: donde se establezcan estrategias y acciones a realizar basado en CRM".

- "Falta de una base de datos que centralice la información actividades operativas y permita gestionar la relación con el cliente:En un modelo CRM, es necesario construir un repositorio, que centralice toda la información operativa", etc. (p. s/n).

\section{Importancia de la investigación}

SEDAPAL (2017) indica que "actualmente, no existe un estudio que haya evaluado y planteado estrategias para las actividades operativas basándose en los fundamentos expuestos por el chatbot que se encuentra dentro del alcance del CRM en la empresa SEDAPAL, y que, además de ello analice sus resultados, y muestre la satisfacción del cliente posterior a dichas estrategias planteadas. Así mismo, la importancia de este estudio también radica en que este modelo se podría replicar según la OTASS (2014) en las otras 49 empresas prestadoras de servicios de saneamiento que existen en el Perú"(p. 8).

\section{REVISIÓN DE LITERATURA Definición del CRM}

León y Rodríguez (2014) proponen una definición de CRM: "es un proceso sistemático para gestionar la relación de iniciación, atención y terminación con el cliente a través de todos los puntos de contactos con él, para maximizar el valor del portafolio de sus relaciones" (p. 83).

\section{Características del CRM}

Según Peláez (2015) mencionó las características más resaltantes en un entorno CRM son las siguientes:

- Automatización de las ventas."Aquí el procesamiento de ventas se configura en aplicaciones tecnológicas".
- Servicio y soporte al cliente. "El CRM otorga una aplicación que facilita una interacción cliente-empresa de un óptimo nivel de servicio. Ello se visualiza a través de los indicadores de medición".

- Servicio de campo. "Esta fase se ejecuta a través de una comunicación efectiva entre los empleados de la empresa y los diferentes tipos de clientes a los cuales se les hace una visita para ofrecerles un servicio más personalizado".

- Automatización del marketing. "El CRM posee y facilita información a las empresas sobre los diferentes hábitos de compra, preferencias y necesidades de los clientes."(p. 253).

\section{Chatbots}

Deloitte digital (2018) mencionó "un chatbot es un agente de software conversacional, que interactúa con los usuarios usando lenguaje natural. Los chatbots ofrecen una experiencia de conversación utilizando inteligencia artificial y procesamiento del lenguaje natural para imitar conversaciones con personas reales"(p.11).

\section{MATERIALES Y MÉTODOS}

Para integrar un chatbot a la estrategia de negocio de SEDAPALpor actividades operativas, se propone la metodología siguiente (Salesforce, 2018):

a. Descubrir lo que se quiere lograr "El área donde se situaeste artículo en SEDAPAL son los Equipos Operación y Mantenimiento de redes - EOMR, tienen como responsabilidad planificar, programar, dirigir, evaluar y controlar las actividades de operación y mantenimiento de redes secundarias de agua potable y desagüe; así como las actividades de reparación y mantenimiento de conexiones domiciliarias de agua potable; y las actividades de limpieza y desinfección de reservorios, cisternas y sus elementos de control hidráulicos; solucionando las emergencias, que se presen- 
ten en el ámbito de competencia, entre otros" (p. s/n).

Las necesidades inmediatas que se propone lograr son:Creación de nuevos canales de atención al cliente por actividades operativas, Mejora de la experiencia de servicio a los clientes y Disminución de costos administrativos como el reclamo, generado por actividades operativas.

El público objetivo, son: i) externos: los clientes, nuevos clientes; e ii) internos: personal de atención al público, áreas operativas (EOMR) y proveedores.

Determinar el objetivo a alcanzar

Zohn (2018) indica que "el objetivo a alcanzar es la Implementación de un chatbot para mejorar la comunicación con los clientes referido a problemas operativos generados en el sistema de red de agua y/o alcantarillado administrado por la empresa SEDAPAL" ( $p$. $s / n)$.

b. Definir la línea de negocio en la que se centrará el chatbot

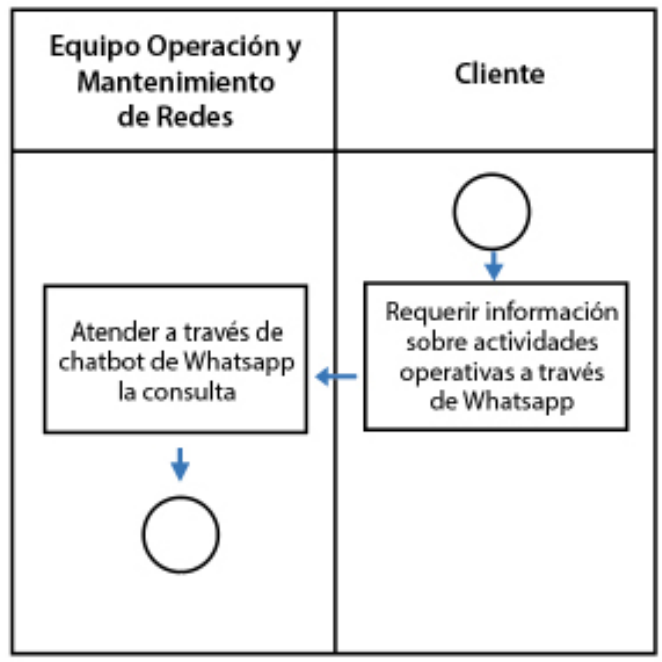

Figura 2. Proceso de comunicación de actividades operativas Imprevistas a través de Whatsapp.
Dada el modelo diseñado el chatbot será atendido B2C: Business to Consumer (siendo la otra línea de negocio como B2B), ya que el negocio se relacionará con los clientes, proponiendo dar una respuesta rápida a consultas como (Colby, 1999):

c. "Trabajo de mantenimiento en tubería", "Falta de agua en predio (casa)", "Fuga de agua en caja de control", "Baja presión (casa)", "Atoro en red y en conexión", "Filtración de desagüe", "Calidad del agua", "Aniego", "Adecuación física de la conexión domiciliaria", "Limpieza a medidor operativo", "Trabajos de mantenimiento inconcluso", etc. (p. s/n).

Los nuevos canales de comunicación con el cliente propuestos podrían ser:Mensajería: Whatsapp, Facebook Messenger; Redes sociales: Facebook; Microblogging: Twitter y Página web.

Y la forma de actuar se muestra en el procedimiento propuesto para actividades operativas imprevistas y programadas.

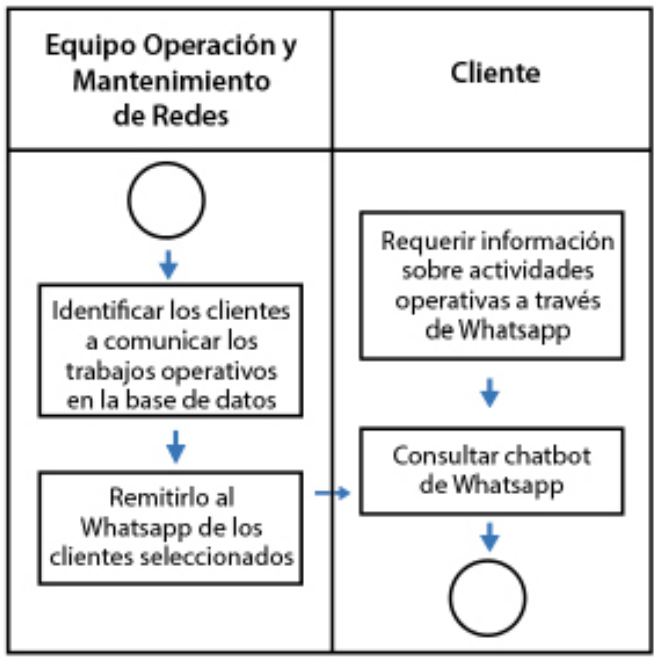

Figura 3. Proceso de comunicación de actividades operativas Programadas a través de Whatsapp. 
d. Definir el tipo de chatbotrequerido Según Guschat (2017) existe en el mercado una diversidad de categorías/tipos de bot, como: bots de utilidades, bots sociales, bots asistentes, entre otros; en este artículo se considerará:

ServiceBot: Con la proliferación de dispositivos móviles y el acceso inmediato a internet los usuarios tienen altas expectativas sobre la atención al cliente. Las personas buscan recibir atención inmediata a través de sus canales preferidos (p. 49).

¿Qué hacen?Los Servicebots están disponibles 24/7 365 días del año, resuelven preguntas frecuentes y realizan operaciones de baja complejidad al instante, mejorando la experiencia de los clientes. Este bot permite la interacción entre cliente y operador a través de una consola de atención híbrida.

\section{RESULTADOS}

Como parte de la investigación realizada en este trabajo, la recolección de la información se efectuó mediante la utilización de la técnica de la Encuesta electrónica, a través de una herramienta informática denominada SurveyMonkey (https://es.surveymonkey.com/).

En ese sentido la encuesta se realizó de la manera siguiente:Tipo de investigación: Cuantitativa; Técnica a utilizar: Encuesta electrónica; Público objetivo: clientes externos y Fecha de realización: Del 20 al 24 de enero de 2020.

Las preguntas planteadas están en función de los objetivos a conseguir y se agruparon en comunicación, interfaz y eficiencia y son: Comunicación:

1. El Whatsapp es el medio de comunicación ideal para comunicarse con la empresa.

2. Le comunicaron a través del chatbot de Whatsapp oportunamente las actividades operativas programadas.

3. Desea que a través del chatbot del Whatsapp se le comparta información relacionada a la empresa.

4. Considera que la encuesta al final de la interacción con el chatbot del Whatsapp es importante.

Interfaz:

5. El chatbot de Whatsapp tiene una interfaz amigable y sencilla para el cliente.

6. La interacción con el chatbot de Whatsapp fue agradable.

Eficiencia:

7. El chatbot de Whatsapp resolvió las dudas del cliente.

8. El chatbot de Whatsapp le da el detalle que requería.

9. Ante una incidencia operativa imprevista, el chatbot de Whatsapp es el mejor asistente para atender su requerimiento.

10. Realizaría transacciones a través del chatbot del Whatsapp.

Luego de realizado la encuesta, realizamos una tabulación de los datos, considerando que la respuesta "SI" es óptimo y "No" es deficiente; evidenciamos que en el tipo "Eficiencia" es donde habría mejorar en esta primera versión del chatbot planteado. 


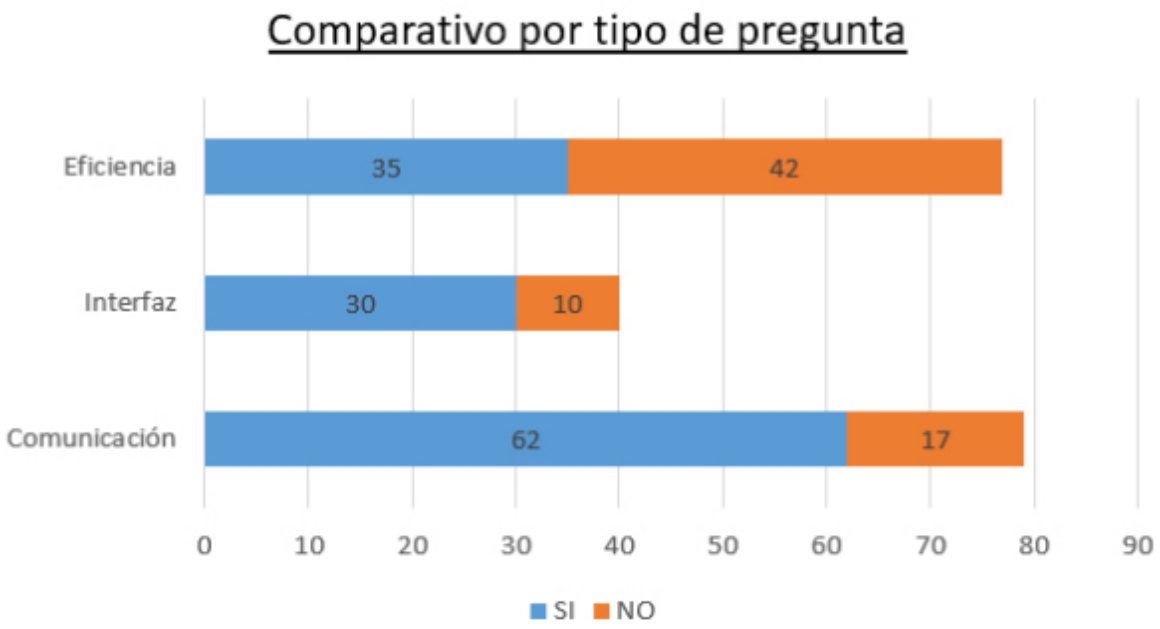

Figura 4. Comparativo de respuesta por tipo de pregunta.

\section{Retorno de la Inversión: ROI}

Para poder comprender la inversión que se va a realizar, es necesario conocer algunos componentes de la Arquitectura para chatbot que se requiere,mostradas en la figura 5.

1. User Interface(s). Se determina la plataforma a utilizar el chatbot, y eso debería ser preguntándose ¿Cómo los clientes accederán al chatbot de manera eficiente?. Por ejemplo, los más utilizados son el website, Facebook Messenger o whatsapp.
2. Digital Assistant.es como está construido el chatbot en su interior, del cual resaltaremos tres aspectos importantes:

2.1 Preprocesamiento

Según Bradbury (2007) un chatbot necesita del NLP de preprocesamiento para manejar los errores típicos.

El NLP es decir la Programación Neuro-Lingüística (Natural LanguageProcessing), describe como descomponerlo, comprender su significado, determinar la acción adecuada y responder en un idioma que el usuario pueda entender.

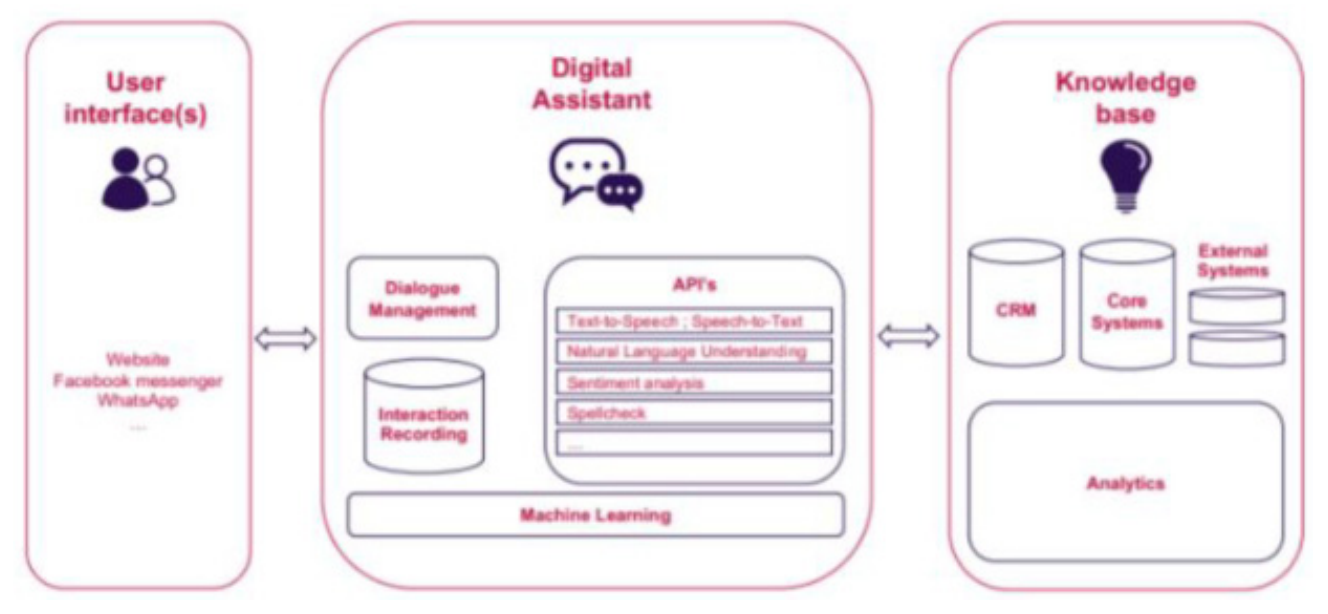

Figura 5. Architecture for public service chatbots Fuente: PwC EU Services (2019) 
Después de realizar el preprocesamiento, el resultado será una lista de oraciones y palabras limpiasdentro de cada oración. El siguiente paso es definir una clasificación.

\subsection{Clasificación}

Cualquier código de clasificación de intención puede cometer errores de dos tipos:

- Falsos positivos: el usuario no expresa una intención, pero el chatbot identifica una intención

- Falsos negativos: el usuario expresa una intención, pero el chatbot no encuentraeso.

Para ello según Sondhi (2008) "se utiliza la Comprensión del Lenguaje Natural (NLU, Natural LanguageUnderstanding), es un subconjunto del NLP que trata con la faceta mucho más estrecha, pero igualmente importante, de cómo manejar mejor las entradas no estructuradas y convertirlas en una forma estructurada que una máquina pueda entender y actuar" (p. s/n).

\subsection{Selección de respuesta}

Una vez que el chatbot comprende el mensaje del usuario, el siguiente paso es generar una respuesta. Una forma es generar una respuesta estática simple. Sondhi (2008) indica que "otra forma es conseguir una plantilla basada en la intención y poner algunas variables. En el desarrollo del chatbotla empresa elige el método para generar la respuesta según el propósitopara el cual se emplea el chatbot" (p. s/n).

3. Knowledge base. Está referido a los diversos repositorios que se tiene para poder comprender y responder mejor al cliente, incluyendo temas de analítica.

Ahora bien, según Phillips (2008) “mencionó a fin de determinar el retorno de la inversión - ROI, por la propuesta planteada aplicamos la siguiente fórmula" (p. s/n):

Respecto a la inversión:

$\mathrm{ROI}=$ Beneficios esperados (acumulados) Total inversion

Para poder implementar "esta propuesta, se requiere de un equipo de trabajo que lo pueda hacer (tiempo del proyecto: 1 mes), así como la suscripción para mantener este servicio anualmente (por el servicio contratado), lo que asciende a la suma de\$26,223.10 dólares. A continuación, se presenta el desagregado de los costos" (Peláez, 2015, p.s/n).

Costo de personal a implementar esta propuesta:
Para lograr implementarlo se debe contratar un Equipo que desarrolle el chatbot, que incluye la administración del proyecto, diseño UX, configuración, pruebas y puesta en producción. Phillips (2008) considera que "este chatbot usará la ProgramaciónNeuro-Lingüística (NLP), Comprensión del Lenguaje Natural (NLU) y la Generación del Lenguaje Natural $(\mathrm{NLG})^{\prime \prime}(\mathrm{p} . \mathrm{s} / \mathrm{n})$.

Tabla 2. Respecto a la inversión

\begin{tabular}{rlr}
\hline $\mathrm{N}^{\circ}$ & \multicolumn{1}{c}{ Descripcion } & Cantidad e Importe \\
\hline 1 & Horas al dia & 8 \\
2 & Dias al mes & 22 \\
3 & Tarifa por hora Jefe de Proyecto & $\$ 18,35$ \\
4 & Tarifa por hora Analista de Sistemas & $\$ 13.57$ \\
5 & Tarifa por hora Programador & $\$ 9.52$ \\
6 & Duracion del proyecto (en meses) & 1 \\
\hline
\end{tabular}

(*) Un dólar equivale a 3.31 soles 
Tabla 3. Costo del personal de desarrollo y configuración

\begin{tabular}{|c|c|c|}
\hline Roles & Horas & $\begin{array}{c}\text { Total } \\
\text { importe } \\
\$\end{array}$ \\
\hline $\begin{array}{l}\text { Jefe del } \\
\text { proyecto }\end{array}$ & 176 & $3,229.15$ \\
\hline Analista & 176 & $2,387.96$ \\
\hline Programador & $\begin{array}{l}176 \\
\text { Total }\end{array}$ & $\begin{array}{r}1,675.99 \\
\$ 7.293 .10\end{array}$ \\
\hline
\end{tabular}

$\left(^{*}\right)$ Un dólar equivale a 3.31 soles

Tarifa de la suscripción:

La tarifa de suscripción de un Chatbot es un pago mensual el cual incluye: a) Número de usuarios activos y de alojamiento; b) Integraciones con el sistema de la empresa, es decir integrar el bot con la infraestructura y las bases de datos de clientes existentes como el CRM;c) Soporte posterior al desarrollo; d) UpgradedeA$\mathrm{Pl}$, e) Procesamiento de Lenguaje Natural (NLP).Siendo entonces la tarifa de suscripción:

Tabla 4. Tarifa de suscripción

\begin{tabular}{|c|c|c|c|}
\hline Descripción & Costo unitario $* *$ & $\begin{array}{c}\text { Cantidad por } \\
\text { mes }\end{array}$ & Total importe \$ \\
\hline Texto o Asistente de Google & $\begin{array}{l}\text { 0,004 USD por } \\
\text { solicitud }\end{array}$ & 70,000 & 280.00 \\
\hline $\begin{array}{c}\text { Entrada de audio } \\
\text { Solicitudes de detección de intent } \\
\text { que incluyan entradas de audio } \\
\text { (reconocimiento o transcripción de } \\
\text { voz). }\end{array}$ & $\begin{array}{l}0,0085 \text { USD por } 15 \\
\text { segundos de audio }\end{array}$ & 35,000 & 297.50 \\
\hline \multirow[t]{3}{*}{ Análisis de opinión } & $\begin{array}{l}\text { De } 0 \text { a } 1 \text { millón de } \\
\text { consultas: } \\
1 \$ / 1000 \text { consultas }\end{array}$ & 1,000 & $1,000.00$ \\
\hline & & Total mensual & \$ $1,577.50$ \\
\hline & & Total anual & \$ $18,930.00$ \\
\hline
\end{tabular}

$\left(^{*}\right)$ Un dólar equivale a 3.31 soles, ${ }^{* *}$ Ediciones de Dialogflow

Respecto al beneficio:

Peláez (2015) "respecto al beneficio se ha considerado que si el 3\% de los reclamos que se presentan no se dieran la empresa ahorraría \$ 5,007.57dólares anualmente" (p. s/n). 
Tabla 5. Respecto al beneficio

\begin{tabular}{lrr}
\hline \multicolumn{1}{c}{ Descripcion } & \multicolumn{2}{c}{ Total importe } \\
& \multicolumn{1}{c}{$\$$} \\
\hline Costo por cada reclamo (1) & $\$$ & 20.51 \\
Incremento promedio de 5 años de cada reclamo $(2)$ & $\$$ & $2,458.30$ \\
TOTAL $(1) *(2)$ & $\$$ & $166,919.12$ \\
Reclamos no deberían producirse anualmente $(3 \%)$ & $\$$ & $5,007.57$ \\
\hline
\end{tabular}

(*) Un dólar equivale a 3.31 soles

En ese sentido, se recuperaría la inversión en el sexto año, tal como se aprecia a continuación:

Tabla 6. Retorno de la inversión

\begin{tabular}{cccc}
\hline Años & $\begin{array}{c}\text { Beneficios } \\
\text { esperados }\end{array}$ & $\begin{array}{c}\text { Beneficios esperados } \\
\text { (acumulados) }\end{array}$ & Inversión \\
\hline 1 & $5,007.57$ & $5,007.57$ & $26,223.10$ \\
2 & $5,007.57$ & $10,015.14$ & $26,223.10$ \\
3 & $5,007.57$ & $15,022.71$ & $26,223.10$ \\
4 & $5,007.57$ & $20,030.28$ & $26,223.10$ \\
5 & $5,007.57$ & $25,037.85$ & $26,223.10$ \\
6 & $5,007.57$ & $30,045.42$ & $26,223.10$ \\
\hline
\end{tabular}

\section{DISCUSIÓN Y CONCLUSIONES}

Según Álvarez (2018) "una encuesta realizada por la empresa CCR en el 2017, en el ámbito de Lima y Callao, a personas de 25 a 60 años de los Niveles Socio-Económico A, B, C y D, determinó al $41 \%$ de los clientes no se les ha comunicado los cortes programados del servicio de agua, siendo el mantenimiento de tuberías el de mayor incidencia $76 \%$, luego la limpieza de tanques, entre otros, generando los mismos clientes soliciten información a través de los diversos canales tradicionales que cuenta la empresa, y al no haber encontrado una solución efectiva y rápida a sus problemas han generado reclamos, por ello la importancia de este trabajo" (p. s/n).

La metodología definida "en cuatro pasos: a) Descubrir lo que se quiere lograr, b) Determi- nar el objetivo, c) Definir la línea de negocio en la que se centrará el chatbot, d) Definir el tipo de chatbot requerido"; permitirá la eficiencia en las actividades operativas a través del uso del chatbot; porque se considera integrar a la estrategia de negocio de SEDAPAL en esta propuesta.

El chatbot está dentro del alcance del Marketing conversacional y trae como beneficios un servicio 24 horas, respuestas instantáneas, quejas resueltas rápidamente, respuestas detalladas, buena interacción, entre otros; porque utiliza la Comprensión del Lenguaje Natural (NLU, Natural LanguageUnderstanding) es un subconjunto del NLP que trata de cómo manejar mejor las entradas no estructuradas y convertirlas en una forma estructurada que 
una máquina puede entender y actuar" (Deloitte digital, 2018, p. s/n).

Para esta propuesta Phillips (2008) "el Retorno de la Inversión - ROI, está definido entre la inversión (costo de personal a implementar la propuesta y la tarifa de la suscripción) y la sumatoria acumulada del beneficio (3\% de los reclamos que se presentan no se darían en la empresa), lo que permitiría ahorrar \$ 5,007.57 dólares anualmente, pudiendo recuperar la inversión en el sexto año" (p. s/n).

\section{REFERENCIAS BIBLIOGRÁFICAS}

AbuShawar, B. and Atwell, E. (2015) ALICE Chatbot: Trials and Outputs. ISSN 2007-9737, Vol. 19. UnitedKingdom.

Álvarez, O., Alvarado, K. y Sánchez, k. (2018). El valor de la Inteligencia Artificial en el Servicio al Cliente EciBot. Colombia.

Anuario Estadístico (2016 y 2017). Estadísticos Comerciales de SEDAPAL y el sistema comercial OPEN SGC. Perú.Recuperado de http://repositorio.SEDAPAL.com.pe/handle/SEDAPAL/27

Áshton, K. (2009). That Internet of Things Thing. ReinoUnido.

Bradbury, A. (2007). DevelopyourNLPSkills. 3ra ed.USA.

Brunetta, H. (2014). Del marketing relacional al CRM. Editorial Todo Management. pp. 82. Buenos Aires, Argentina.

Colby, K. (1999). Human-computer conversation in a cognitive therapy program.In Wilks, Y. (ed.) Machine conversations, Kluwer, pp. 9-19.

Croxatto, H. (2005). Creando valor en la relación con sus clientes. Editorial Dunken. Buenos Aires, Argentina.

Deloitte digital (2018). Chatbots Point of View. Deloitte Artificial Intelligence. Fondo Social Europeo. pp. 11. EEUU.
Ediciones de Dialogflowhttps://cloud.google.com/dialogflow-enterprise/?hl=es-419

Guschat (2017). ChatbotsQué son y por qué están revolucionando el comercio digital. España.

Kepuska, V. and Bohouta, G. (2018).Nextgeneration of virtual personal assistants (Microsoft Cortana, Apple Siri, Amazon Alexa and Google Home).Published in: 2018 IEEE 8th Annual Computing and Communication Workshop and Conference (CCWC). USA. DOI: 10.1109/CCWC.2018.8301638

León, J. y Rodríguez, R. (2014) La administración de la relación de los clientes (CRM), una herramienta para crear estrategias competitivas. pp. 83. México.

McFarland, S. (2019).Take Control of Siri 1.0. Editing Sawyer Mcfarland.ISBN 9781947282353. USA.

OTASS (2014). Resultados del Ranking Gobernabilidad y Gobernanza. Perú.

Pastor, LI. (2018). Briefingpaper: los chatbots en educación. pp. 7. UniversitatOberta de Catalunya, España.

Peláez, M. (2015). Desarrollo de componente software en sistemas ERP-CRM.Edición: 5.0. Editorial Elearning S.L. pp. 253.España.

Phillips, J. and Zuñiga, L. (2008).Costs and ROI. Evaluating at the ultimate level.ISBN: 978-07879-8721-3. USA.

PwC EU Services (2019).Architecture for public service chatbots. EuropeanCommission.

Salesforce. (2018). State of chatbotsreport. EEUU.

SEDAPAL (2016). Plan Estratégico Institucional 2017-2021. Perú.

SEDAPAL (2018). Memoria Anual. Perú. 
SEDAPAL. (2017). Informe de Sostenibilidad 2017. pp. 11. Perú.

SEDAPAL. (2017). MORG de SEDAPAL. Aprobado mediante RGG N. ${ }^{\circ}$ 347-2016-GG del 12 de octubre de 2017. pp. 100. Perú.

Sondhi, B. (2008). SpringerHandbook. SpeechProcessing. USA.

Sunass (2007). Reglamento General de Reclamos de usuarios de servicios de saneamiento aprobado a través de la Resolución de Consejo Directivo No 066-2006-SUNASS-CD y publicado el 14 de enero d 2007, y sus modificatorias.

Weizenbaum, J. (1966). ELIZA-A computer program for the study of natural language communication between man and machine. Communications of the ACM, Vol. 10, No. 8, pp. 3645.

Zohn, S. (2018).For God's Sake: Work by the Book!.pp. 129. EEUU.

\section{CORRESPONDENCIA:}

Dr. Jorge Rafael Diaz Dumont

jorgediaz@unat.edu.pe 